\title{
A Measuring and Evaluation Program for the Application of the Ring-Core or Hole-Drilling Method
}

\author{
Andreas $\mathrm{Nau}^{1, \mathrm{a}}$, Berthold Scholtes ${ }^{1, \mathrm{~b}}$ \\ ${ }^{1}$ Institute of Materials Engineering - Metallic Materials, University of Kassel, \\ Mönchebergstr. 3, 34125 Kassel, \\ anau@uni-kassel.de, bscholtes@uni-kassel.de
}

Keywords: hole-drilling, ring-core, integral method, differential method, software, modeling

\begin{abstract}
There is a strong need for reliable residual stress measurements. On the one hand, residual stresses can be beneficial, when they are adapted to external loads. On the other hand, they can be detrimental, when they are unknown. Thus, their occurrence can lead to an uneconomical oversizing of components or in their failure, as well. Apart from diffraction methods, mechanical methods are well recognized in order to determine unknown residual stress states. Depending on the applied method, specific boundary conditions have to be taken into account. In the case of mechanical methods like the hole-drilling and the ring-core method, the characteristics of the geometry of the component should be in accordance with an ideal and thick plate. The reason behind is the need for a calibration data set to transform strains into stresses. The calibration is usually carried out numerically. For the sake of simplicity, the geometry of the component is an ideal thick plate and the hole is introduced in its center. However, in most cases, this is not identical with the geometry of the component under investigation. Hence, an application tool was designed that enables the parametric design of a Finite Element Model, the determination of calibration coefficients, the evaluation of the experiment and the visualization of the results for geometries of practical importance. So far, the application tool can represent plates variable in their geometries and in positioning of the point of measurement. The option for other geometries are also possible e.g. a turbine blade.
\end{abstract}

\section{Introduction}

It is well known that residual stresses (RS) play in important role in various scientific and engineering fields. Introducing and controlling specific residual stress states are a prerequisite for competitive and reliable goods. An impressive example is a turbine blade for gas turbines [1] which are usually shot peened at its blade root. Shoot peening introduces compressive RS states near the surface which is highly recommended for preventing crack growth. This technique is also applied for many parts in more common products, like crankshafts etc. In this context, the hole-drilling method (HDM) and the ring-core method (RCM) stand for cost-effective methods to determine RS. They are classified as semi-destructive mechanical methods [2] and are widely used. Apart from a final report, such analyses involve the execution of the experiment (application of the strain gauge, the removing of the material, etc.), the evaluation of the experimental data and the illustration of the results. However, the most important part is often hidden or unknown for the operator, the numerical calibration (CAL). Thus, a complete application tool should contain all these steps to provide more robust and reliable RS analyses. Indeed, a large number of complete principal RS depth distribution analyses within depths of half of the hole depth or ring-core diameter can be conducted in approximately a few hours with such an application tool.

\section{Basic concept}

RS analyses with the HDM or RCM always call for data which are based upon a CAL. The CAL is necessary due to the fact that there is no analytic strain-stress transformation for blind holes. Thus, a hole-drilling measurement is even more than the experimental part. Fig. 1 schematically shows a complete RS analysis for mechanical RS measurements. On the one hand, it is emphasized 


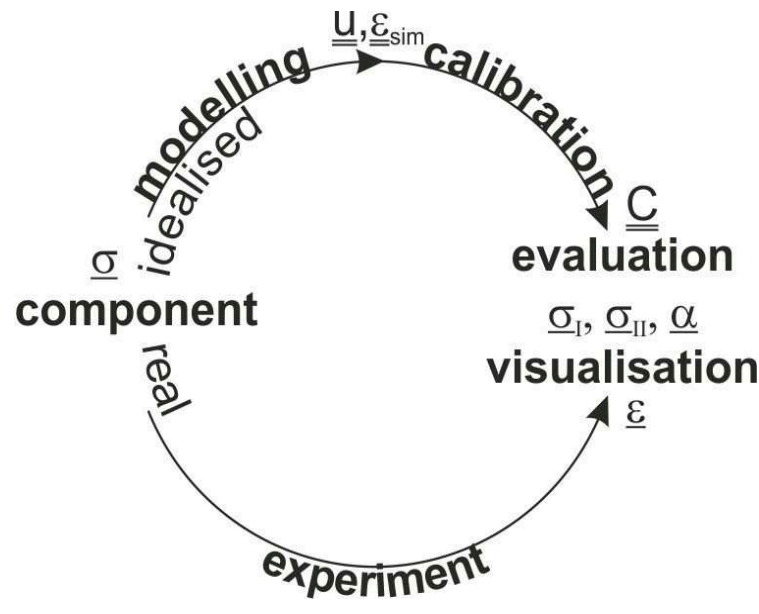

Fig. 1: Process of a semi destructive RSmeasurement. that the component is considered twice: Namely, in the experiment and in an idealized state. The idealized state is used for the CAL which is usually carried out numerically. Finally, the data from the experiment and from the simulation converge at the evaluation step. On the other hand, it is apparent that many intermediate steps are required to obtain the final result, e.g. the access to strains from the simulation $\left(\underline{\mathcal{E}}_{\text {sim }}\right)$ and the experiment $(\underline{\mathcal{E}})$, the determination of the CAL coefficients $\underline{C}$ etc.. Thus, a complete solution is needed.

Modelling. The main advantage by using numerical simulation techniques is an exactly known RS state $\underline{\sigma}_{i j}$. However, many issues are simplified, e.g. the

hole is usually ideally cylindrical and placed in the center of the strain gauge rosette (SGR) and in the center of a thick plate which is not the case in practical experiments. If a commercial evaluation program is used in order to determine the principal RS state $\left(\underline{\sigma_{I}}, \underline{\sigma_{I I}}\right.$ and $\left.\underline{\alpha}\right)$ from experimental strains, the implemented CAL data are strictly speaking restricted to such idealized boundary

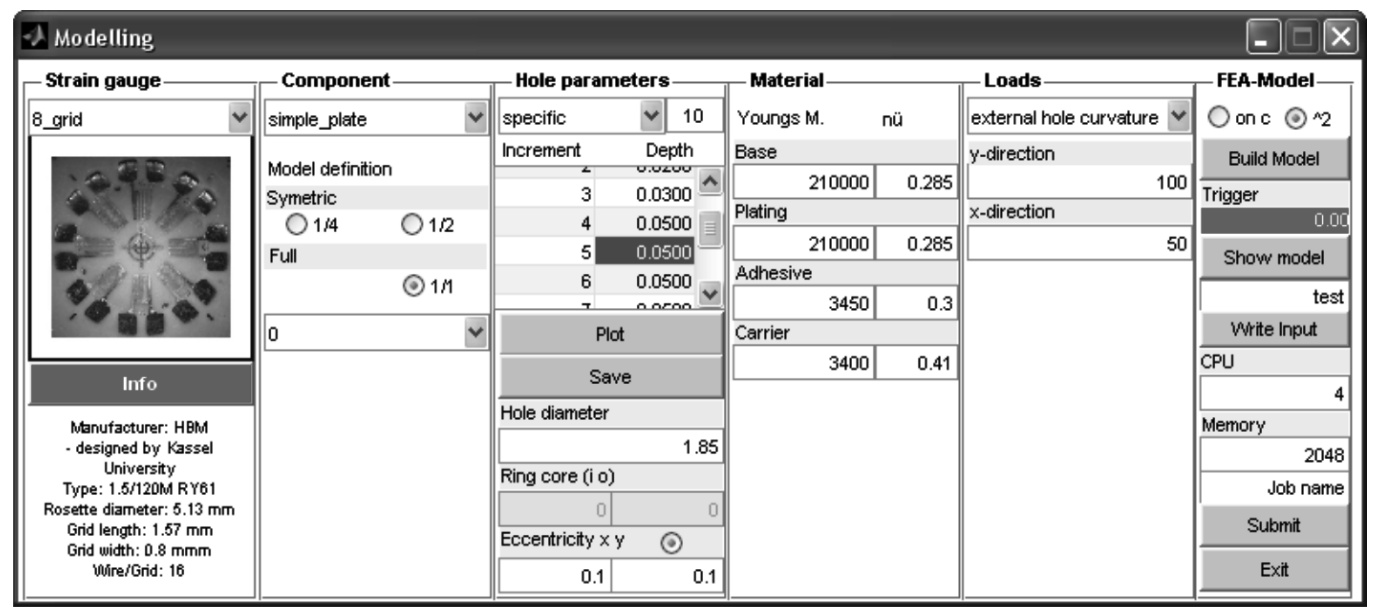

Fig. 2:

Interface for Abaqus to built up finite element models.

conditions. As simple as it sounds, in fact, carrying out a numerical simulation is time-consuming and special. Good skills in modeling and programming are required. Thus, an interface for the Finite Element (FE) program Abaqus was designed to built up a numerical model which is flexible in a lot of parameters, Fig. 2.

With the menu block "Strain gauge", the corresponding strain gauge rosette (SGR) can be chosen. Up to now, three SGR for the HDM are taken into account, Fig. 3a). In addition, the commercially available SGR for the RCM is also implemented. The menu block "Component" supports the parameterization of the component, Fig. 3b). Currently, a plate is available which is arbitrary parametrizable in its dimension and in the location of the point of measurement. The option of considering symmetric conditions can also be taken into account for more economic numerical simulations. In addition, an ideal cladding can be considered.

The Menu block "Hole parameters" provides options to design the incremental steps for the hole, e.g. smaller steps near the surface and larger steps for deeper increments. Furthermore, the hole diameter and its eccentricity to the strain gauge center can exactly be adapted corresponding to the real experiment.

With the menu block "Material", elastic isotropic material properties can be allocated to the bulk component which can differ from the material of the cladding. 
a)

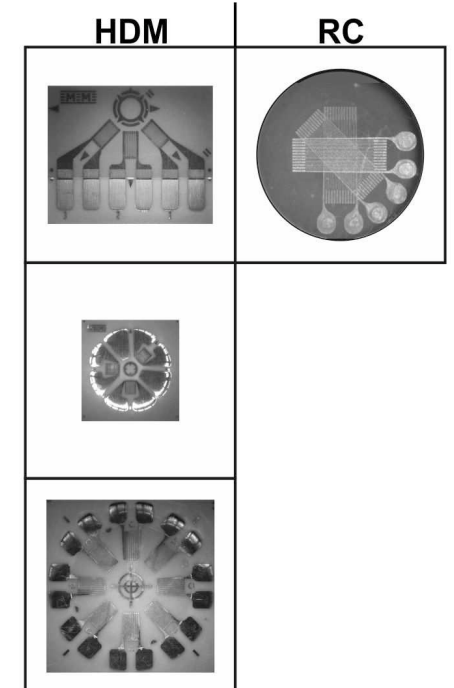

b)

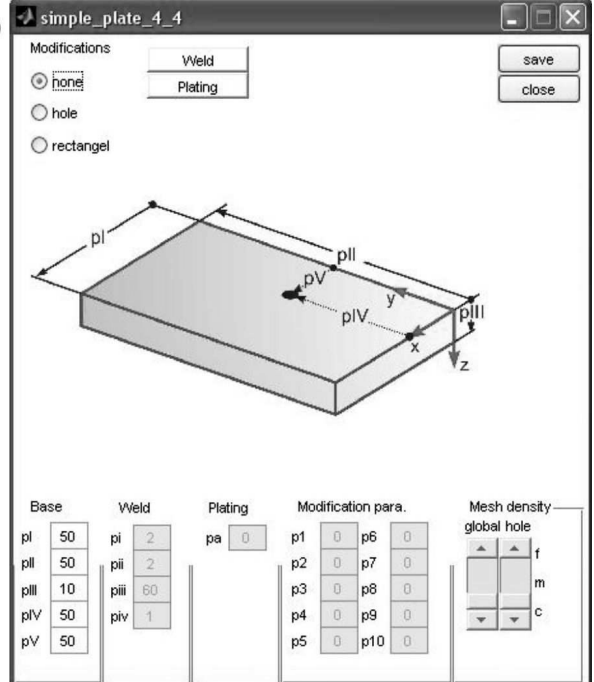

Fig. 3: Geometries of a) the strain gauge rosettes (HDM: CEAXX-UM-XXX (MM), EA-XX-RE-XXX (MM), custom design by Kassel University (HBM); RC: 1-RY51-5/350 (HBM)), b) the component.

The menu block "Loads" supports two options, namely, applying CAL loads on the hole curvature or on the external surfaces of the component. The former is necessary to determine CAL coefficients for the Integral Method (IM), the latter is appropriated for the Differential Method (DM) or for a comparison with an experimental CAL by using applied uniform stress state with a tensile machine. In [3,4] the DM in combination with the eight-grid SGR was used to evaluate various drilling techniques and effects of eccentricity.

a)

b)

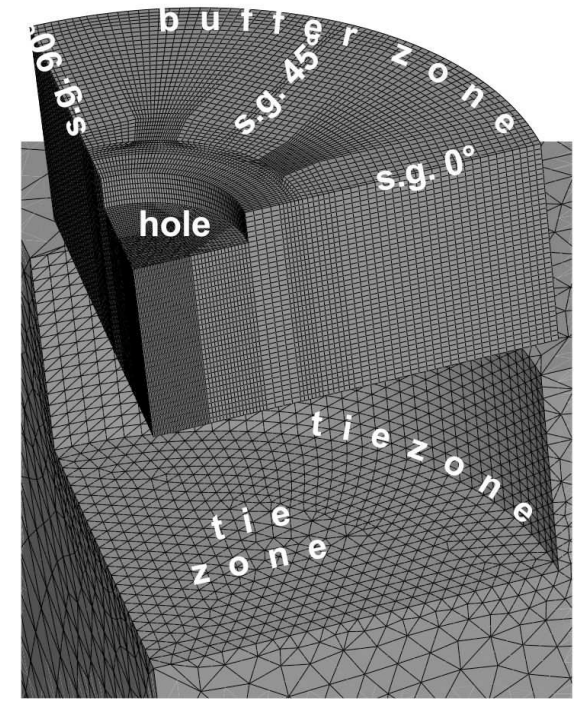

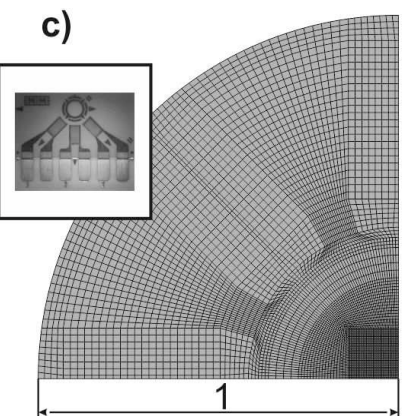
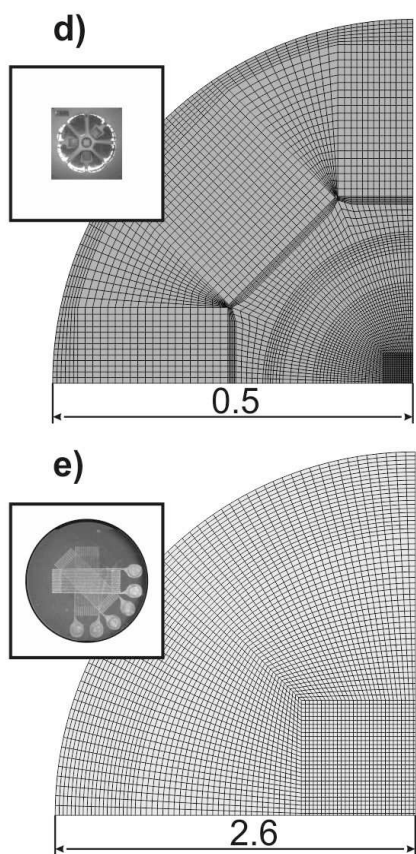

Fig. 4: Finite element model - a) tied components, b) sub and bulk component, c) sub component CEA-XX-UM-XXX, d) EA-XX-RE-XXX, e) 1-RY51-5/350.

Finally, with the menu block "FEA-Model" the complete model is built up with AbaqusCAE by interpreting a python script in just a minute, Fig. 4. The last step is similar to the work presented in $[5,6]$. However, the model itself is based upon another philosophy. The model is composed of a global component automatically meshed with quadratic tetrahedral elements (C3D16R). This strategy is chosen as a simplification to avoid extensive partitioning in case of more geometrically detailed components. Unlike the global component, the sub component is discretized much more detailed by linear (or optional quadratic) hexahedral elements (C3D8R or C3D20R). The sub component considers the geometry of the hole and the SGR. Both meshes are connected by ties. A buffer szone is implemented in the sub component in order to overcome discontinuities in results between both meshes. The hybrid mesh 
was tested in different ways e.g. compared with a reference mesh completely consisting of linear hexahedral elements. The results demonstrate that the hybrid mesh provides a nearly identical accuracy with the advantage of very much faster computation time. The strain gauges were designed with the same space between the filaments like the real case. The released strains were determined by using the displacement method according to [7]. A comparison between double integration of the strains and the strategy mentioned above led to negligible differences.

Determination of calibration coefficients. After completing the numerical simulation, CAL coefficients for the IM and for DM have to be determined according to [2] and [7], respectively. Fig. 5 shows the CAL interface for the IM (the one for the DM is not shown here). The interface accesses the result file of the simulation and reads out the displacements of the strain gauges and the CAL stresses of the removed material. Based upon these data, CAL coefficients are automatically determined and stored as an ASCII-file which is implemented in a database.

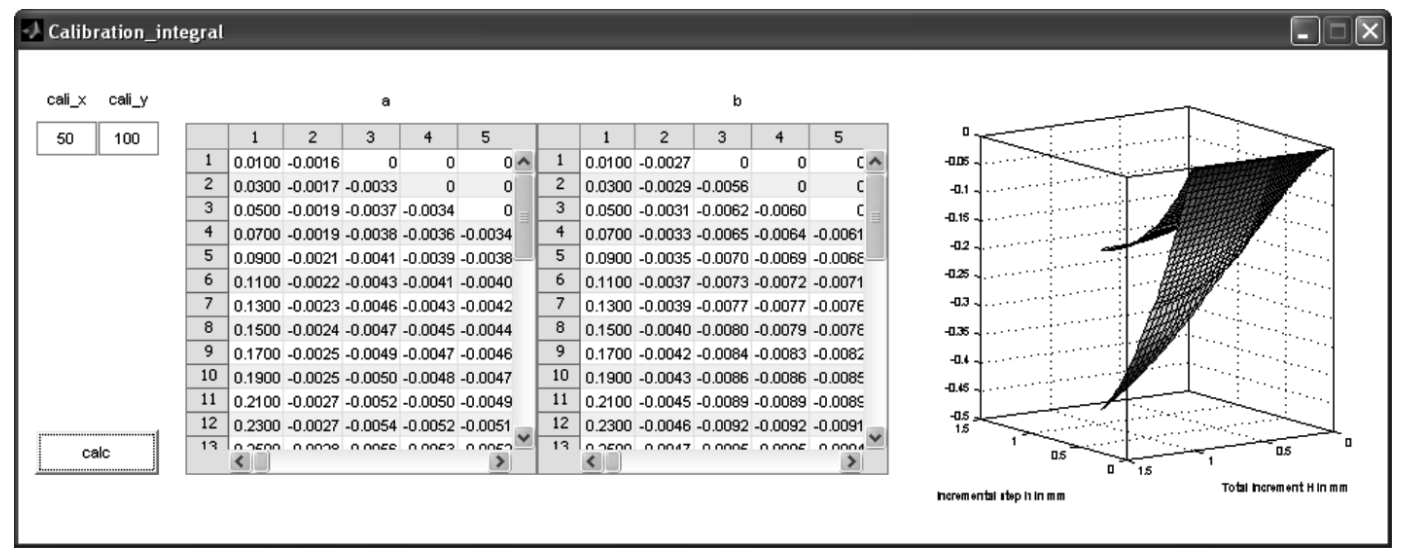

Fig. 5: Module for determining calibration coefficients with the Integral Method.

Experimental evaluation. The experimental conditions can be specified by a user data interface, Fig. 6. The operator has to select the used strain gauges, the thickness of the component, the incremental steps of the material removal process, the chamfer of the bit, the material properties and the evaluation method (IM or DM). Based upon this information, the evaluation of the experiment can be continued, Fig. 7. The experimental strains are automatically imported. Outliers have to be identified and further decisions are required for the need of data conditioning. For this purpose, the modul provides a broad range of data conditioning techniques (average mean value, cubic and
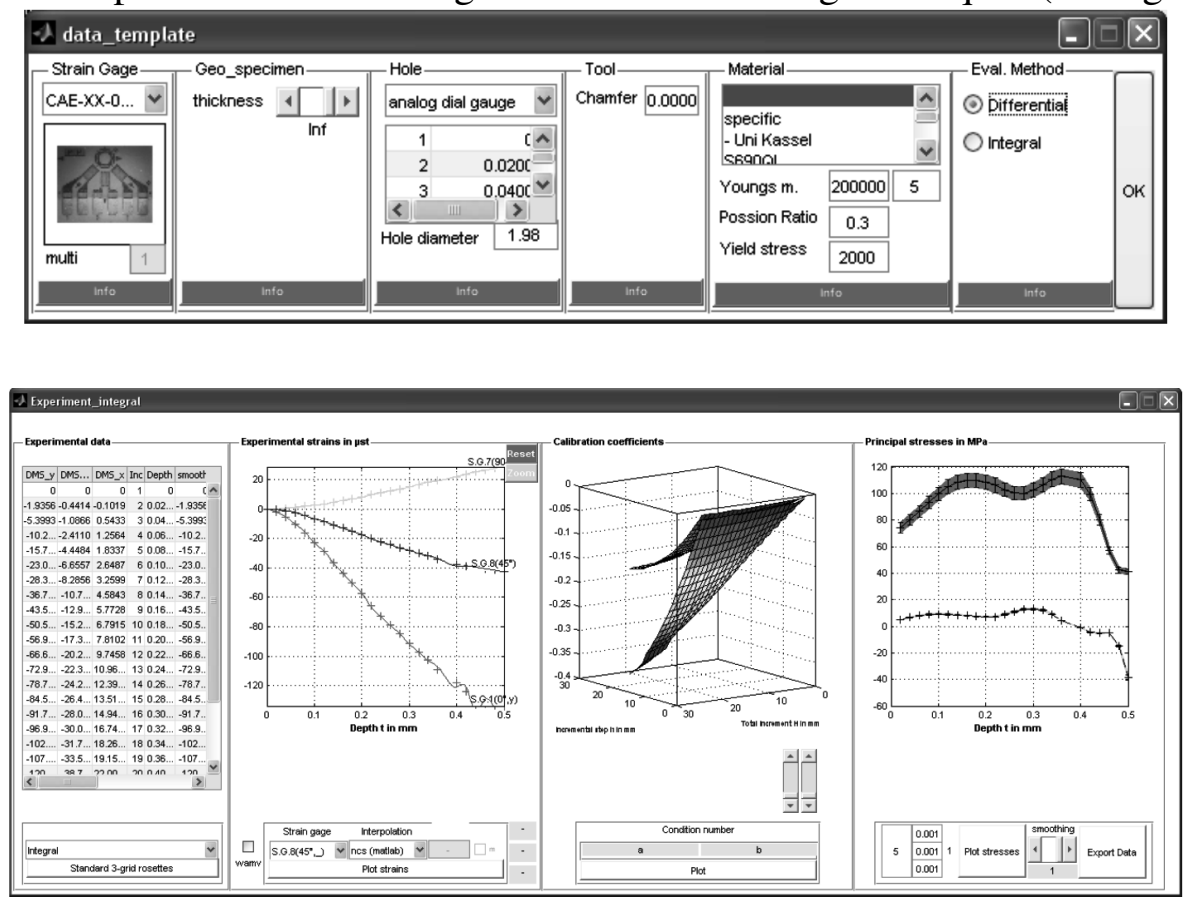

Fig. 6: Module for entering specific experimental parameters.

Fig. 7: Module for evaluating experiments. 


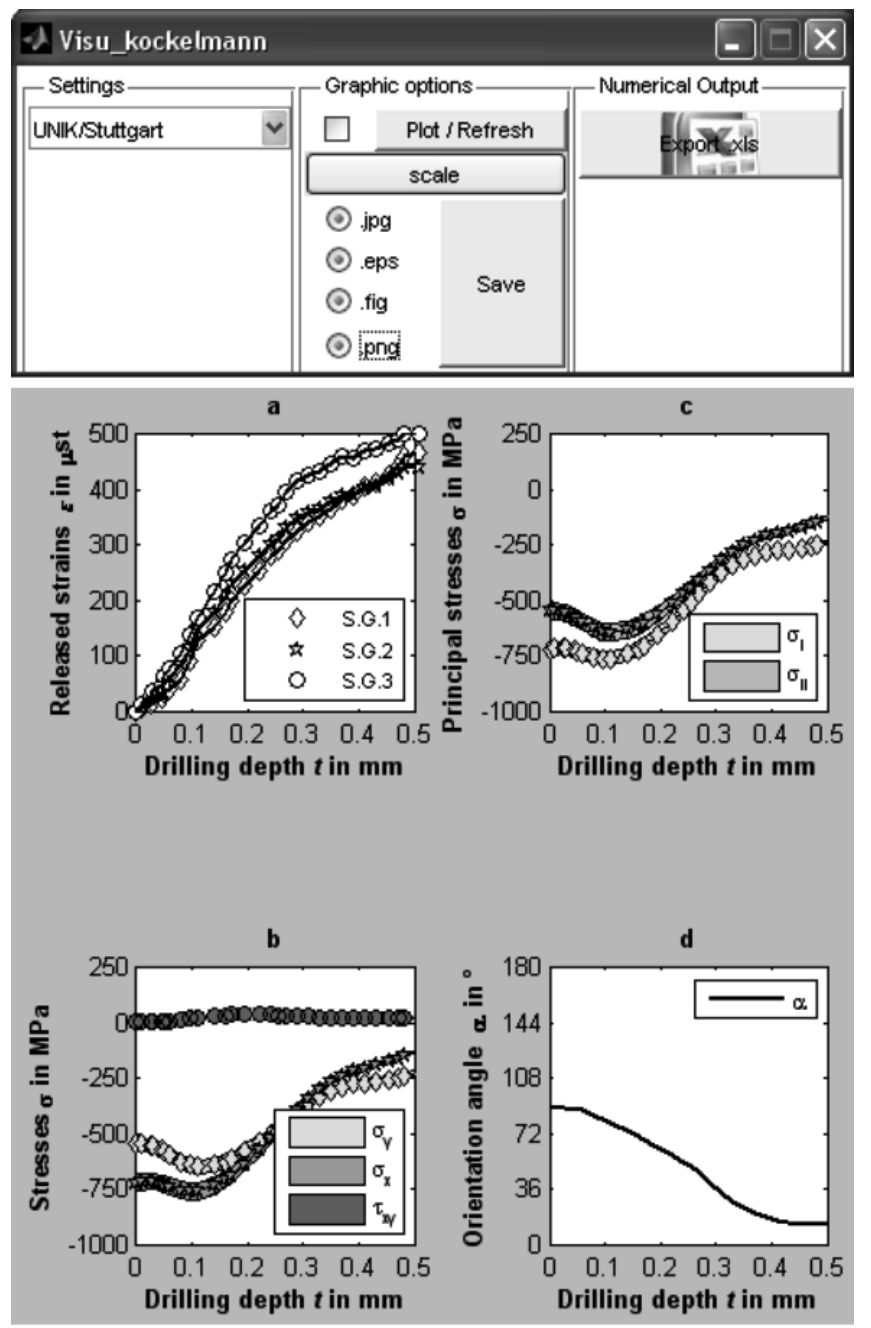

Fig.8: Visualization modul.

smoothing splines, or polynomials). Afterwards, the CAL coefficients are adapted by interpolation of the depths of the experimental increment steps and are imported for the calculation of the stress state. The complete stress state, the input data and the parameters for conditioning are exported in an ASCII-file.

Visualisation of results. Finally, the visualization modul supports the summary of the results by access to the exported ASCII. Four graphs are plotted which display the released strains, the determined stresses according to the strain gauge directions of the used SGR, the resulting principle stresses and the orientation angle of the principle stress state. Each graph can arbitrarily be scaled and finally stored in different file formats. In addition, an excel-sheet can be exported, Fig. 8.

\section{First numerical studies}

Fig. 9 shows first findings of a numerical study which was conducted by using the presented application tool. The study focuses on the impact of a varying plate thickness (th $=1 \mathrm{~mm}$ to $7 \mathrm{~mm}$ ) and a varying Possion Ratio ( $v=0.2$ and 0.5$)$. Hence, models that are different in thickness and material properties need to be built up. This can be carried out in a few hours depending on the mesh quality by using the analysis tool. The findings emphasize that the CAL coefficients are independent of the plate thickness if it is larger than $4 \mathrm{~mm}$. The influence of the Possion ratio is also apparent.

\section{Conclusion and outlook}

Complete application tools are needed for residual stress analysis with the hole and the ringcore method on the one hand and for the research of the method itself on the other hand (e.g. to determine the impact of geometrical boundary conditions, etc.). The proposed application tool is very much flexible in order to built up finite element models, to provide a complete environment to calibrate results taken from numerical simulations, to evaluate and reporting experimental data. It is planned to investigate more geometrical effects like the influence of the edge of the component etc. and to support data transfers for arbitrary platforms.

\section{Acknowledgement}

The authors gratefully acknowledge the support of Federal Ministry of Economic Affairs and Energy, which enabled the detailed investigation of the hole-drilling and the ring-core Supported by: method.

on the basis of a decision by the German Bundestag 


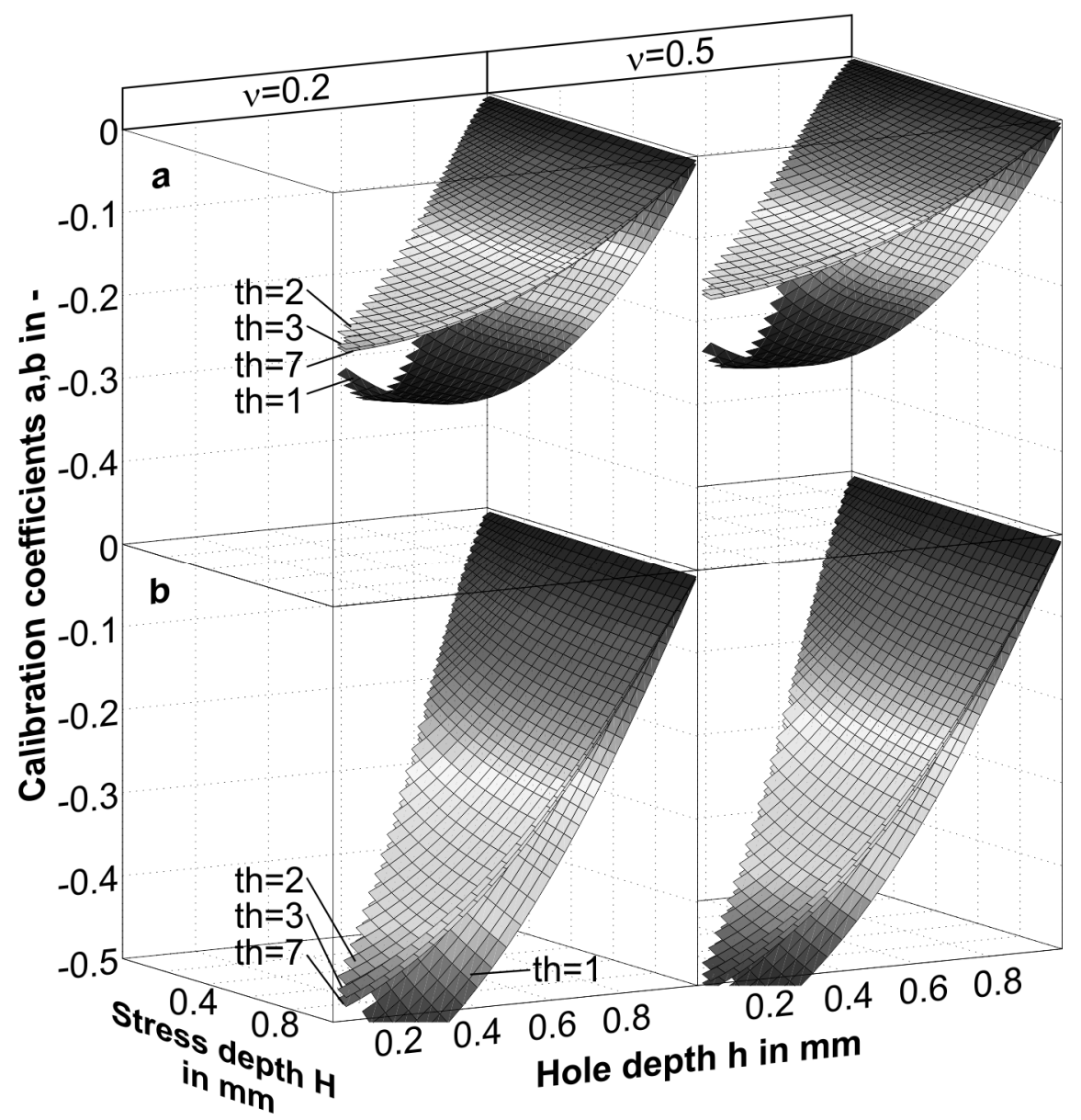

Fig.9: Influence of the thickness of the plate and the Possion ratio.

\section{References}

[1] Feldmann, G. G. (2012) Verbesserte mechanische Oberflächenverfestigung von Schaufeln an integral beschaufelten Hochdruckverdichterrotoren. Dissertation.

[2] ASTM E837-08 (2008) Standard Test Method for Determining Residual Stresses by the Hole-Drilling Strain Gage Method.

[3] Nau, A., Scholtes, B. (2013) Evaluation of the High-Speed Drilling Technique for the Incremental Hole-Drilling Method. Experimental Mechanics 53(4):531-542.

[4] Nau, A., Scholtes, B. (2012) Experimental and Numerical Strategies to Consider Hole Eccentricity Experimental and Numerical Strategies to Consider Hole Eccentricity for Residual Stress Measurement with the Hole-Drilling Method with the Hole Dri lling Method. Materials Testing 54(5):296-303.

[5] Civin, A., Vlk, M. (2009) Theoretical Analysis of Ring-Core Method for Residual Stress Determination. Conference ANSYS(2009).

[6] Xiao, B., Li, K., Rong, Y. (2009) Numerical Study on Calibration Coefficients for Holedrilling Residual Stress Measurement. Proceedings of the SEM Annual Conference.

[7] Schwarz, T. (1996) Beitrag zur Eigenspannungsermittlung an isotropen, anisotropen sowie inhomogenen, schichtweise aufgebauten Werkstoffen mitrels Bohrlochmethode und Ringkernverfahren. Dissertation, University of Stuttgart. 